\title{
Article \\ The Anomalous Behavior of Thermodynamic Parameters in the Three Widom Deltas of Carbon Dioxide-Ethanol Mixture
}

\author{
Evgenii Igorevich Mareev ${ }^{1,2, * \mathbb{D}}$, Alexander Petrovich Sviridov ${ }^{1}$ and Vyacheslav Mihailovich Gordienko ${ }^{1,2}$ \\ 1 Federal Scientific Research Centre "Crystallography and Photonics", Institute of Photon Technologies, \\ Russian Academy of Sciences, Pionerskaya St. 2, Troitsk, 108840 Moscow, Russia; \\ sviridoa@gmail.com (A.P.S.); v_m_gord@mail.ru (V.M.G.) \\ 2 Faculty of Physics, M. V. Lomonosov Moscow State University, Leninskie Gory Bld. 1/2, \\ 119991 Moscow, Russia \\ * Correspondence: mareev.evgeniy@physics.msu.ru
}

Citation: Mareev, E.I.; Sviridov, A.P.; Gordienko, V.M. The Anomalous

Behavior of Thermodynamic

Parameters in the Three Widom Deltas of Carbon Dioxide-Ethanol Mixture. Int. J. Mol. Sci. 2021, 22, 9813. https://doi.org/10.3390/ ijms22189813

Academic Editor: Dongho Kim

Received: 8 July 2021

Accepted: 8 September 2021

Published: 10 September 2021

Publisher's Note: MDPI stays neutral with regard to jurisdictional claims in published maps and institutional affiliations.

\begin{abstract}
Using molecular dynamics, we demonstrated that in the mixture of carbon dioxide and ethanol ( $25 \%$ molar fraction) there are three pronounced regions on the $\mathrm{p}$-T diagram characterized by not only high-density fluctuations but also anomalous behavior of thermodynamic parameters. The regions are interpreted as Widom deltas. The regions were identified as a result of analyzing the dependences of density, density fluctuations, isobaric thermal conductivity, and clustering of a mixture of carbon dioxide and ethanol in a wide range of pressures and temperatures. Two of the regions correspond to the Widom delta for pure supercritical carbon dioxide and ethanol, while the third region is in the immediate vicinity of the critical point of the binary mixture. The origin of these Widom deltas is a result of the large mixed linear clusters formation.
\end{abstract}

Keywords: supercritical fluids; mixture; molecular dynamics; clusters; Widom delta

\section{Introduction}

In recent years, supercritical fluids (SCF) have become the object of close attention due to their wide applications in technological processes of purification, separation, and extraction of various substances, in such areas as biotechnology, food industry [1], propulsion systems [2], liquid-propellant rocket engines, high-pressure gas, nanoparticle and cluster production [3,4], and supercritical chromatography [5]. Significant progress has been achieved in understanding supercritical fluids' physical and chemical properties [6,7], primarily due to improved experimental techniques [8,9]. However, several questions regarding the physical micro- and macroscopic behavior of supercritical fluids remain open. In the phase diagram, the line separating the liquid from the gas phase ends in the critical point. Previously, it was believed that the supercritical state is homogeneous. Recent experiments identified areas with different liquid or gaseous properties even under supercritical conditions [10]. Indeed, above the critical point in the p-T diagram, a change in the structure of the substance is also observed: from weakly interacting molecules and their clusters (gas-like SCF) to "superclusters" with "interspersed" regions of almost empty space (liquid-like SCF) [11-14]. The change in the SCF structure from liquid-like to gas-like occurs during passing through the coexistence frontier, separating noticeable changes in the state of the medium. This so-called Widom delta (due to the delta-like shape on the p-T diagram) was first identified experimentally by K. Nishikawa and I. Tanaka [10]. They demonstrated the difference of the supercritical state from the classical representation as having homogeneous properties. The Widom region is usually associated with the thermodynamic transition of a single-component system. T. Sciortino et al. [15] introduced the Widom region as a set of states with the maximum correlation length [7,16]. Still, often this area is approximated as the locus of points on the p-T diagram, where the maximum thermodynamic parameters are reached since they can be more easily estimated [17-20]). 
From the known results [21], it follows that impurities shift the location of the Widom delta in supercritical $\mathrm{CO}_{2}$ [22] and $\mathrm{H}_{2} \mathrm{O}$ fluids [23]. Such investigations were carried out for several individual substances, such as $\mathrm{CO}_{2}$ [24-28], $\mathrm{O}_{2}$ [29], $\mathrm{Ar}$ [7,30], and $\mathrm{H}_{2} \mathrm{O}$ [31], as well as mixtures of noble gases. Crucially, that the addition of even a tiny amount of another substance with different critical pressure and temperature can significantly shift the critical parameters of this system (up to hundreds of degrees and bars), because in a first approximation, the critical temperature and the critical pressure of the resulting mixture can be estimated as partial values of the critical parameters of each component. In addition, the presence of two components in the system significantly complicates the interaction between molecules and particles. This interaction ensures the appearance of mixed clusters and anomalous behavior of thermodynamic (TD) parameters. In our previous paper [32], we revealed that, in the $\mathrm{CO}_{2}$-ethanol mixture, large mixed clusters are formed, which leads to the increase of nonlinear refractive index $\left(n_{2}\right)$. The effect of anomalous $n_{2}$ increase could find applications in nonlinear optics. However, there are still a lot of open questions, primarily regarding the impact of clusters on the macroscopic properties and the whence of the Widom delta. Nowadays, there is a gap in describing the thermodynamics of transition states in multicomponent systems in addition to identification of various phases. The range of existence of the Widom delta for binary mixtures is of great technological importance. In particular the $\mathrm{CO}_{2}$-ethanol mixture is applied in the process of cannabis extraction [33], fish oil extraction [34], caffeine extraction [35], etc. The increase of solubility that is achieved in the Widom delta (due to clustering) can significantly increase the efficiency of these processes [36]. It is also worth mentioning that another substance can be initially dissolved in ethanol, and due to the effective diffusion of SCF carbon dioxide, the substance then spreads throughout the system [37]. Therefore, it is important to predict the range of optimal pressures and temperatures for extraction, ablation, nanoparticle production, etc.

In this work, we have expanded the well-proven molecular dynamics methods (MD) [38] to study the behavior of the thermodynamic parameters of a carbon dioxide and ethanol mixture (density, enthalpy, specific isobaric heat capacity), as well as the microstructure of the medium (parameters of clustering of the medium) in the Widom delta vicinity.

\section{Results and Discussion}

Before discussing numerical simulation results, it is necessary to recall that a mixture of carbon dioxide with ethanol in a molar ratio of 3:1 under room conditions is a two-phase system $[39,40]$. In such a mixture, liquid ethanol with a small amount of dissolved carbon dioxide molecules in it is surrounded by gaseous carbon dioxide. MD calculations show that at temperatures below critical for carbon dioxide (for example, $275 \mathrm{~K}$ ) and atmospheric pressure, carbon dioxide dissolves well in ethanol (the calculated enthalpy of mixing is of the order of $-4 \mathrm{~J} / \mathrm{mol}$, which is comparable with the tabular $-3.7 \mathrm{~J} / \mathrm{mol}$ [41], the free energy of mixing is about $-1.5 \mathrm{~J} / \mathrm{mol}$ ). Figure $1 \mathrm{a}$ also shows a uniform distribution of ethanol and carbon dioxide molecules. A significant amount of carbon dioxide molecules (about $50 \%$ ) is dissolved in ethanol, forming a quasi-homogeneous mixture. Another part of the molecules is in the gas phase outside the formed supercluster. Below the critical pressure and temperature, with an increase in temperature at constant atmospheric pressure, the isolated structure (the dissolved in the ethanol $\mathrm{CO}_{2}$ ) begins to pass into a gaseous state. With an increase in pressure up to about 40 bar (at room temperature), the mixture turns into a liquid state [42]. However, the transition to the supercritical state of matter significantly complicates the microstructure of the mixture. In the case of a one-component system (pure $\mathrm{CO}_{2}$ ), the single Widom delta is achieved (see Section 3). In this region, a maximum of density fluctuations is reached, and abnormal behavior of thermodynamic (TD) is observed. Moreover, in this region, the liquid-like SCF transforms into a gas-like SCF. 

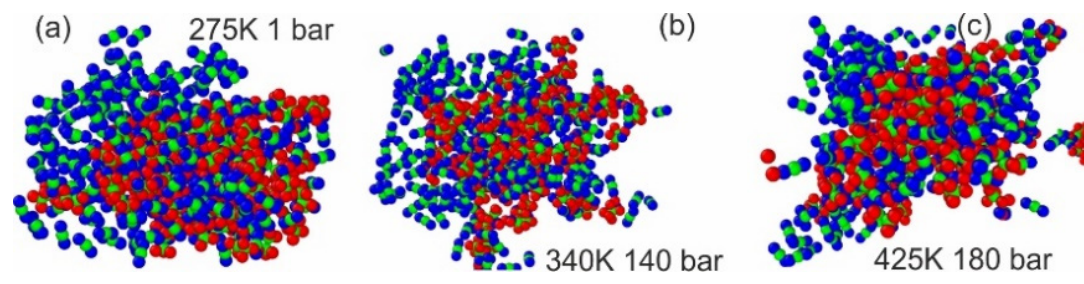

(d)

Figure 1. Visualization of the simulation results. Molecules of carbon, oxygen, and hydrogen are shown in green, blue, and red correspondingly. The figure shows the values of temperature and pressure. (a) Liquid ethanol with dissolved carbon dioxide with surrounding gaseous carbon dioxide; (b) the Widom delta of carbon dioxide; (c) the most compressed mixture with a uniform distribution of carbon dioxide and ethanol molecules; (d) the Widom delta of the mixture.

As we showed in [32], supercluster decay is observed in the Widom delta, which is characteristic of a liquid state of matter and a liquid-like SCF. Therefore, at the first stage, the MD method was used to study the dependence of the medium density $(\rho)$ and the maximum clusters size $\left(\mathrm{S}_{\max }\right)$ on pressure and temperature. In this work, the Widom region was identified as a region with maximum density fluctuations, accompanied by the disintegration of the supercluster.

For pure carbon dioxide, the Widom region can be identified by determining the maximum dependence of the partial density derivative on pressure for different temperatures. As shown in Figure 2, on the heat map of the density derivative on pressure $\delta \rho / \delta p$ $(p, T)$, there is a region where the maxima of this derivative are observed. This graph was constructed based on the data [43], with the following numerical differentiation. The physical processes associated with the Widom delta (an extremum of thermodynamic parameters, maximum density fluctuations, and cluster formation) are most clearly manifested near the critical point. At a longer distance, the experimental conditions on the $\mathrm{p}-\mathrm{T}$ (pressure-temperature) diagram from the critical point, the peak of density derivative on pressure becomes wider (Figure 2) and its amplitude is decreased. The maximal amplitude is achieved for a pressure of $79 \mathrm{bar}$ and a temperature of $308 \mathrm{~K}$.

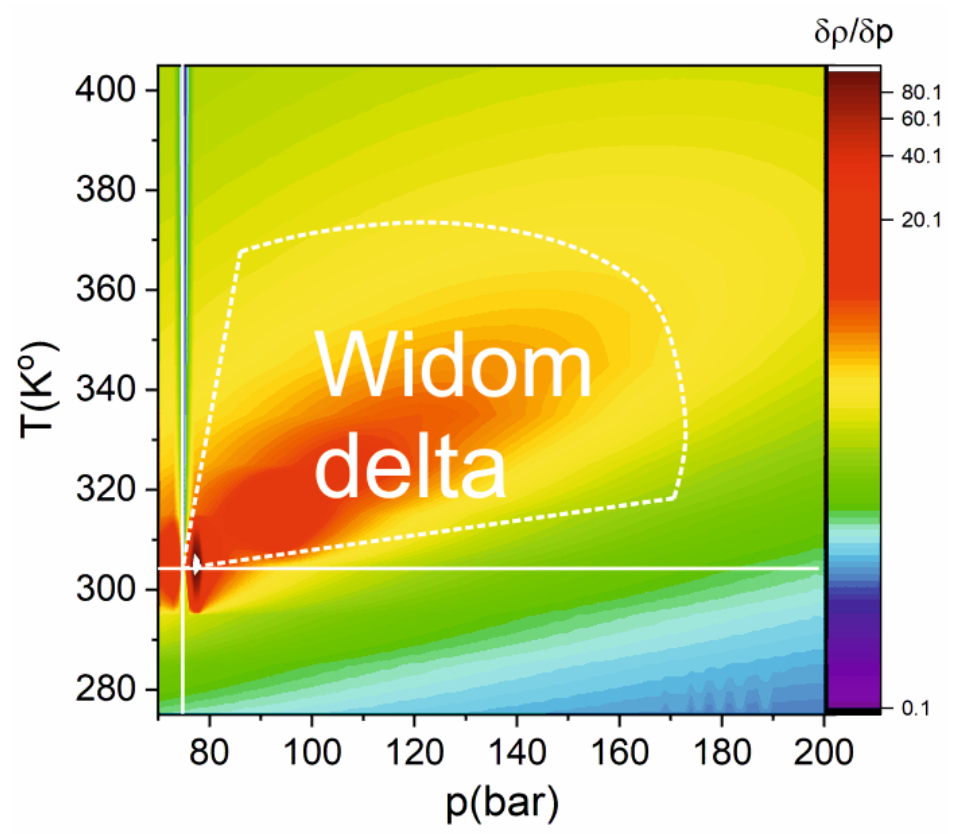

Figure 2. Heat map of the density derivative on pressure $\delta \rho / \delta p$ (logarithmic scale) in $\mathrm{CO}_{2}$. The dashed white line shows the Widom delta [43].

Figure 3a shows heat maps of the mixture density dependence on pressure and temperature. The local density was calculated using Radical Voronoi tessellation, which was previously successfully applied for characterization of the gas-like and liquid-like 
SCF [44]. The tessellation was performed with Voro++ library [45]. Several pronounced areas can be distinguished in this figure. The first region corresponds to a two-phase medium; it is in the region of relatively low pressures (less than 40 bar) and temperatures (less than $400 \mathrm{~K}$ ), see Figure 1a. At higher pressures, most of the mixture is in the liquid phase. However, in the vicinity of $310 \mathrm{~K}$ at pressures above $80 \mathrm{bar}$, a decrease in the mixture density is observed (a solid curve marks the area in Figure 3a). Furthermore, in the vicinity of $410 \mathrm{~K}$, a local density maximum is observed, and in the region of $420-460 \mathrm{~K}$, a rapid (2.5 times) decrease in density is observed at pressures above 60 bars. The Widom delta of carbon dioxide is observed for temperatures about $310 \mathrm{~K}$ and a pressure of about 80 bar. Similar behavior is also observed for other thermodynamic (TD) parameters.
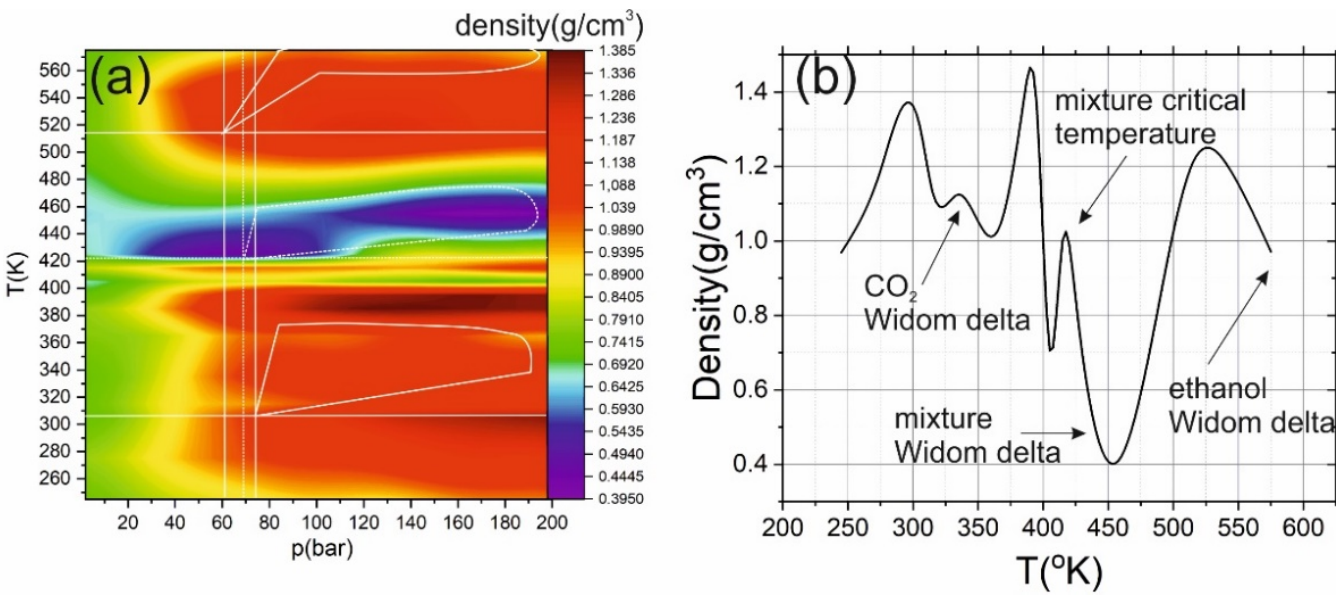

Figure 3. (a) Heat map of the mixture density dependence on pressure and temperature. The highlighted areas correspond to the Widom delta (solid lines for carbon dioxide and dashed lines for mixtures). (b) Dependence of density on temperature from Figure 1a under pressure of 161 bar. The solid lines show the critical temperatures and pressures of carbon dioxide and ethanol; the white dashed line shows the mixture critical point.

The maximum of calculated cluster size $\left(\mathrm{S}_{\max }\right)$ equals to 9260 molecules. It is achieved in the region of maximum density. In this region, the structure of the mixture is close to that of a liquid (i.e., infinite supercluster [46]). As a result, most molecules from one massive cluster called a supercluster. The Widom delta corresponding to pure carbon dioxide is visible in Figure 4. It shows a decrease in the maximum size of the supercluster by about $1 / 3$ (from 9260 to 6030 molecules). However, this region is less pronounced than the Widom region of the mixture (in the vicinity of $420 \mathrm{~K}$ ). In the area with the maximum cluster size, carbon dioxide and ethanol form one dense structure with an almost uniform distribution of ethanol molecules over the system's volume (see Figure 1c), forming a homogeneous medium. In the Widom delta of carbon dioxide, $\mathrm{CO}_{2}$ molecules form separate clusters (see Figure 1) containing only $\mathrm{CO}_{2}$ molecules. Due to the intermolecular interaction between the mixture components (in high pressure, more than 80 bar), some carbon dioxide molecules remain in the supercluster, and the clustering process does not develop as efficiently as in pure $\mathrm{CO}_{2}$. It is essential to note the area shown by the dashed curve in Figure 4. In the microstructure of the system corresponding to this region, we can find large linear mixed clusters (see Figure 1d). As we revealed in [28], the formation of linear clusters leads to the anomalous behavior of the optical and nonlinear optical properties of the medium in the Widom delta. The Widom delta corresponding to ethanol is less pronounced than the Widom delta corresponding to carbon dioxide or the mixture due to weaker interaction between ethanol molecules. In addition, only $25 \%$ of ethanol is in the mixture, and secondly, most of the interactions occur between different molecules. 

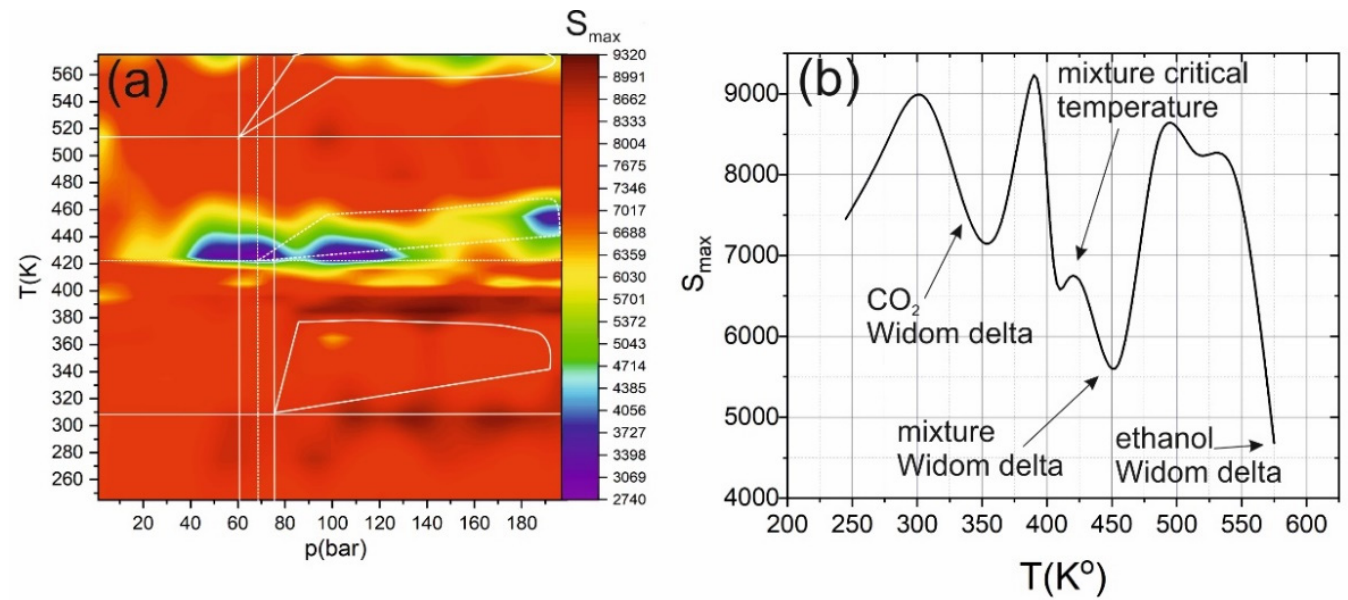

Figure 4. (a) Heat map of the maximum cluster size $S_{\max }$ (number of molecules) dependence on pressure and temperature. The solid lines show the critical temperatures and pressures of carbon dioxide and ethanol; the white dashed line shows the mixture critical point. The highlighted areas correspond to the Widom delta (solid lines for carbon dioxide and dashed lines for mixtures). (b) Dependence of the maximal cluster size on temperature under pressure of $161 \mathrm{bar}$.

Since the Widom delta was initially defined as the region in which fluctuations reach a maximum [47], density fluctuations were retrieved using molecular dynamics. The procedure of fluctuations calculation was performed based on the atomic volume occupied by each molecule (which gives the values of the local density). This value was averaged; the variance was calculated and subsequently normalized to the average density value. A three-dimensional heat map of density fluctuations is shown in Figure 5. It shows that fluctuations have the lowest values in the liquid phase (pressure above 80 bars, temperature below $320 \mathrm{~K}$ ). There is also a region of a gas-like supercritical state, at a pressure above 60 bars and a temperature higher than $480 \mathrm{~K}$. The most increased fluctuations (more than twice the average values) are achieved in the Widom delta for the mixture (pressure over 60 bars, temperature over $420 \mathrm{~K}$ ) and in transition from two-phase system to single-phase system (curve starting at $20 \mathrm{bar}$ and $260 \mathrm{~K}$ and ending at 100 bar and $325 \mathrm{~K}$ ). The Widom delta of individual components of the mixture is less pronounced: in the region of $340 \mathrm{~K}$ and 80 bars for carbon dioxide, and $560 \mathrm{~K}$ and 100 bars for ethanol.
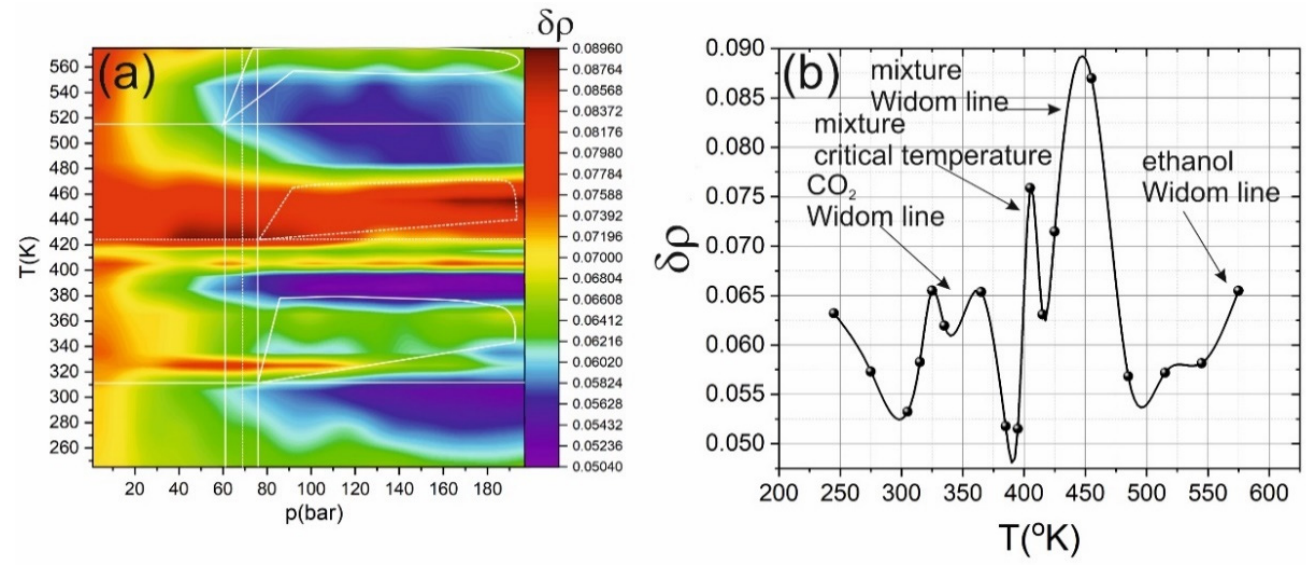

Figure 5. (a) Heat map of the density fluctuations $\delta \rho / \delta p$ dependence on pressure and temperature. Solid lines show the critical temperature and pressure of carbon dioxide and ethanol; dashed lines show mixture parameters. The highlighted areas correspond to the Widom delta (solid lines for carbon dioxide and dashed lines for mixtures). (b) The dependence of density fluctuations $\delta \rho$ on temperature from Figure $5 \mathrm{a}$ under pressure of 161 bar. 
It should be noted that fluctuations in the gas phase are more intense than in the liquid one. The rise of fluctuations is due to the relatively weak interaction between individual molecules, which leads to the appearance of local inhomogeneities in the system. Density fluctuations are primarily associated with the microstructure of the medium when, due to the formation of mixed clusters, regions with both high and low density are formed in the medium (see Figure 1d). If we consider the Widom delta as a region separating the liquid-like SCF from the gas-like SCF, then it is here that an intermediate case is realized, for which the coexistence of the liquid-like SCF (cluster-regions of increased density) with the gas-like SCF (rarefaction region) is characteristic. Growth of density fluctuations (in comparison with pure media) will lead to increased solubility and more efficient solvation processes.

In contrast to a sufficiently rapid change in the fluctuations of the mixture density with varying temperature and pressure, the difference in enthalpy occurs more smoothly, see Figure 6. The enthalpy has several local extrema; the first corresponds to the transition of ethanol to the gas phase, the second to the Widom delta of the mixture. Outside these zones, the dependence of enthalpy on temperature and pressure is monotonic. It is worth noting here a region with a rapid decrease in enthalpy in the vicinity of $400 \mathrm{~K}$ at a pressure of more than $60 \mathrm{bar}$, which also corresponds to a reduction in the enthalpy of mixing (from $\sim-4 \mathrm{~J} / \mathrm{mol}$ at room temperature and atmospheric pressure to $\sim-8 \mathrm{~J} / \mathrm{mol}$; the free energy of mixing is on the order of $-3 \mathrm{~J} / \mathrm{mol}$ ). This sharp decrease does not correspond to any Widom delta (neither the mixture nor the individual components). However, in this region, the best mixing of carbon dioxide with ethanol is achieved (see Figure 1c).

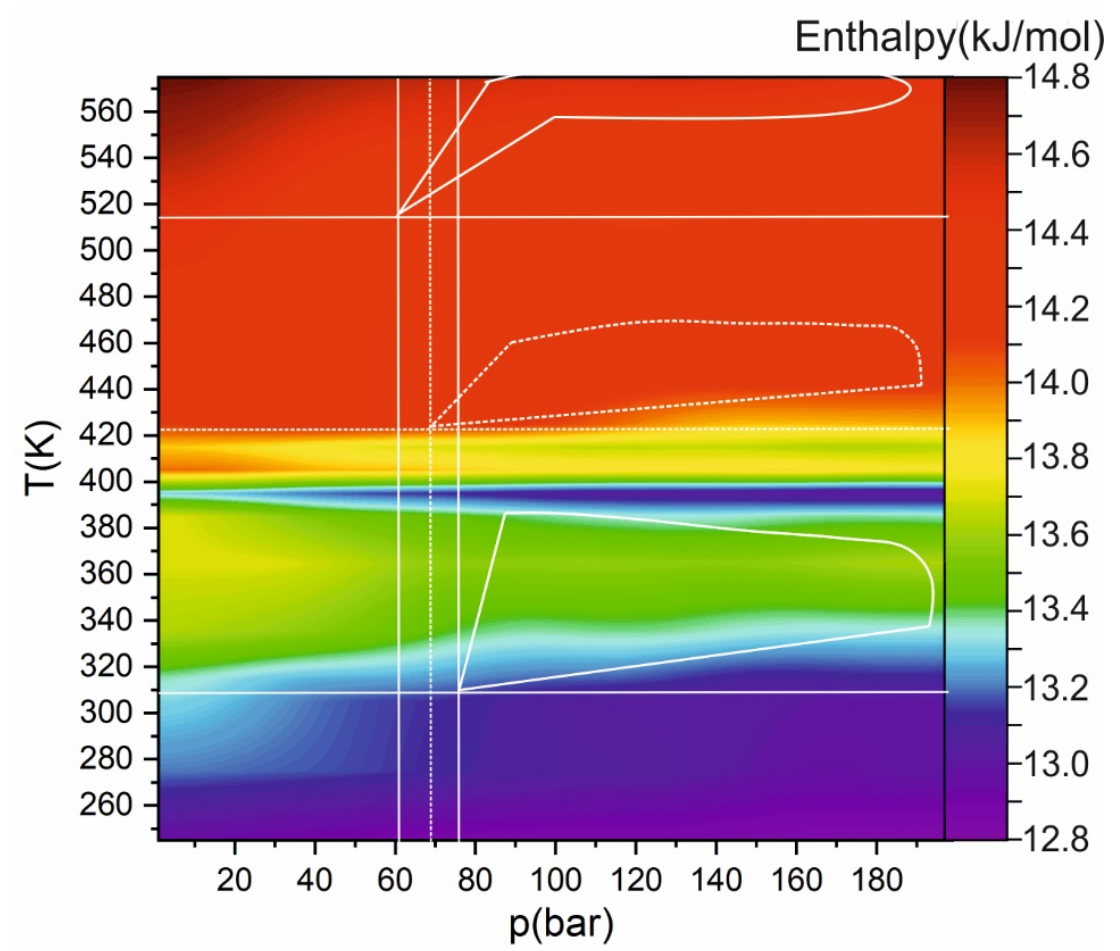

Figure 6. Heat map of enthalpy dependence on pressure and temperature. Solid lines show the critical temperature and pressure of carbon dioxide and ethanol; dashed lines correspond to critical mixture parameters. (Solid lines for carbon dioxide and dashed lines for mixtures.)

In contrast to enthalpy, its first derivative (specific isobaric heat capacity) has a much more complex behavior, reflecting all changes in the structure of matter: a transition to a supercritical state, a transformation from a single-phase to a two-phase system, a transition from a liquid-like to a gas-like SCF, etc. (see Figure 7). 


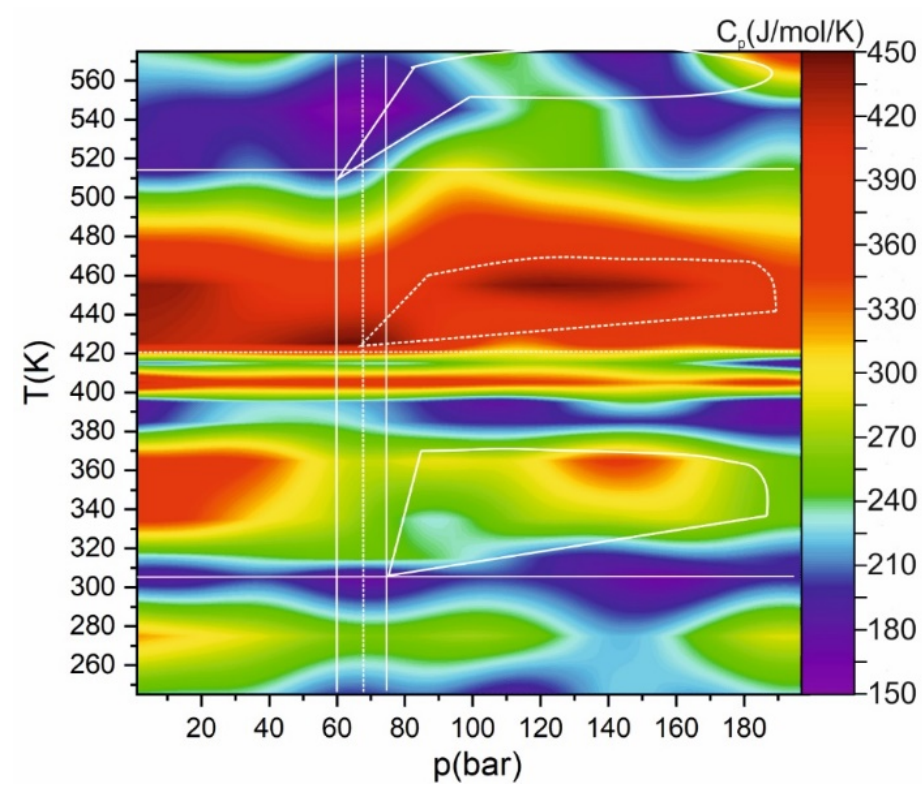

Figure 7. Heat map of specific isobaric heat capacity $C_{p}$ on pressure and temperature. Solid lines show the critical temperature and pressure of carbon dioxide and ethanol; dashed lines show critical mixture parameters. The highlighted areas correspond to the Widom (solid lines for carbon dioxide and dashed lines for mixtures).

The performed numerical simulation precedes the experimental study of the specific properties of the binary mixture under investigation using optical techniques that are simple, non-invasive, and sufficiently accurate. Such optical parameters of a medium as linear and nonlinear refractive indices depend on the nature of clustering of the medium [26]. Moreover, with an increase in the number of clusters of medium size with a pronounced axis, the molar refraction and the value of the nonlinear refractive index growth, as was previously shown on the example of pure carbon dioxide [26]. Thus, the observed appearance of large linear mixed clusters should lead to a sharp change in the medium's optical properties. Change in the parameters of the medium also leads to a modification of the vibrational spectrum of the mixture. It can be verified, for example, by the method of Raman spectroscopy [48]. The growth of fluctuations in the Widom region can also be retrieved by measuring the laser beam intensity fluctuations transmitted through a medium [49]. Based on the results of the performed simulation, it is possible to schematically depict the observed Widom regions on the $\mathrm{p}-\mathrm{T}$ diagram (see Figure 8). The growth of fluctuations increase the diffusion inside the media and can also enhance the rate of multiple chemical reactions or increase their efficiency [50]. However, the applied model does not describe the possible chemical reaction between the components, similarly to the chemical reaction between supercritical methanol and carbon dioxide on the inner surface of the cell [51]. 


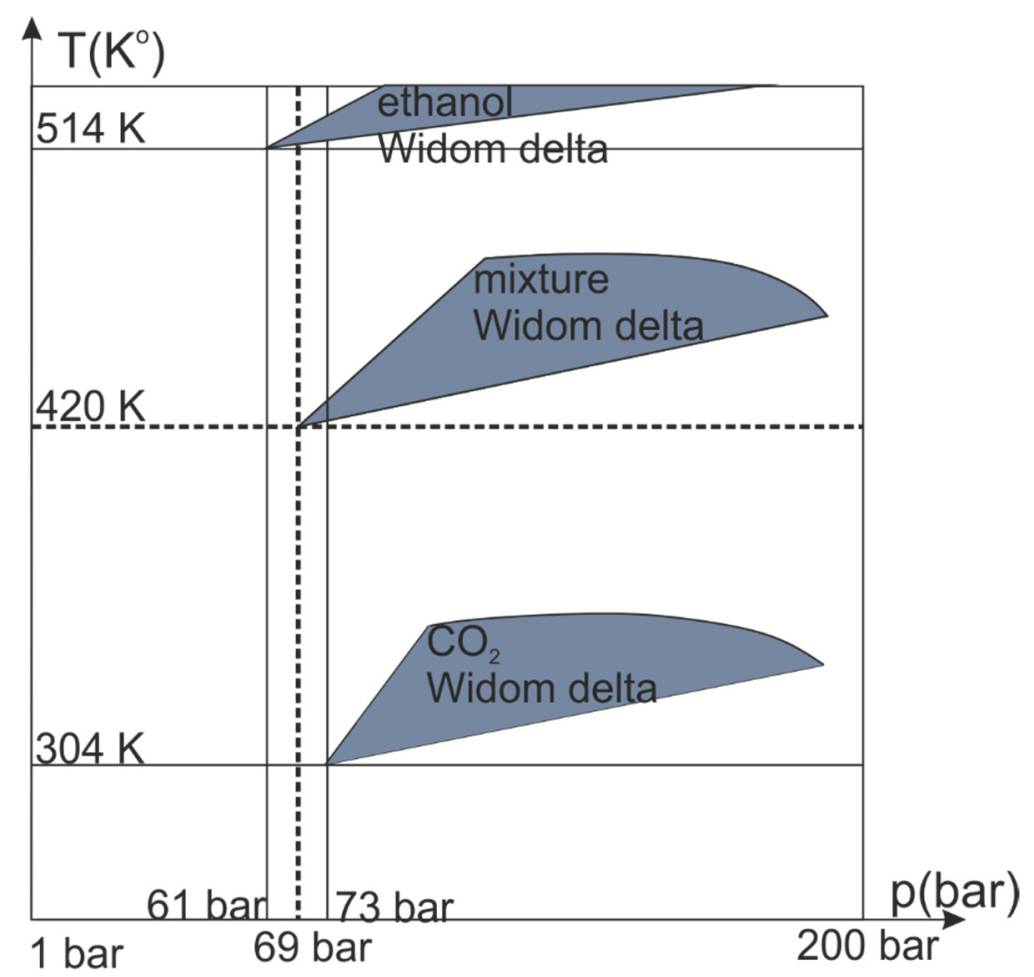

Figure 8. Schematic representation of a pressure-temperature diagram for a $\mathrm{CO}_{2}-\mathrm{C}_{2} \mathrm{H}_{5} \mathrm{OH}$ mixture. The dark regions show three Widom delta. Solid lines show the critical temperature and critical pressure for individual components. The dashed line shows critical temperature and critical pressure for the entire mixture.

The three observed Widom regions are expected to exist for several mixtures of linear molecules. The alignment of linear clusters is primarily due to a nonzero dipole moment in the ethanol molecule and a quadrupole moment in the carbon dioxide molecule. Thus, in the Widom region, they form extended linear clusters. In symmetric molecules (such as $\mathrm{SF}_{6}$ ) or noble gases $(\mathrm{Ar}, \mathrm{Ne}, \mathrm{Xe})$, the effect may also appear but will be less pronounced. The formation of mixed $\mathrm{CO}_{2}-\mathrm{C}_{2} \mathrm{H}_{5} \mathrm{OH}$ clusters results from intermolecular interactions and is not limited to the investigated mixture. The complex mixtures can open a new route for new optical materials or efficient solvents. For instance, the nonlinear refractive index in the mixture Widom delta is triple of $\mathrm{CO}_{2}$ at the same pressure, due to the increase of form-factor $\gamma$ (anisotropy factor) of linear clusters up to 0.8 ( 0.7 in pure $\mathrm{CO}_{2}$ Widom delta) [25]. It is also important to note that, formally, the Widom delta corresponding to the $\mathrm{CO}_{2}$ component lies below the critical point on the p-T diagram (see Figure 8). Such a behavior arises from formation of pure $\mathrm{CO}_{2}$ clusters. In this Widom delta, the mixture demonstrates properties similar to supercritical fluid such as high-density fluctuations and cluster formations. In a contrast, under temperatures above the critical point the maximal fluctuations and cluster formations only achieved in the mixture's Widom delta. Therefore, the "formal" transition to the supercritical state does not guarantee the appearance of high fluctuations and cluster formation, and vice versa, the high fluctuations and cluster formations could be observed outside supercritical state.

In the development of the topic under discussion, alcohols such as ethanol or methanol are suitable solvents. Therefore, the use of deuterium-containing alcohols will allow organizing a mixture, including another substance (for example, liquid $\mathrm{D}_{2} \mathrm{O}$ ) that can be initially dissolved in ethanol. Due to the effective diffusion of SCF carbon dioxide, the substance then spreads throughout the system, forming a monophase medium. This approach will allow us to perform experiments on the implementation of generating a neutron flux with the $\mathrm{MeV}$ energy level because of the action of super-intensive (more than $10^{17} \mathrm{~W} / \mathrm{cm}^{2}$ ) femtosecond laser radiation on deuterium-containing aggregates [52]. This approach's 
essence consists of the production of cluster jets during the supersonic expansion of supercritical matter into a vacuum for subsequent use as intense laser targets. Currently, the production of cluster jets during the supersonic expansion of a supercritical mater into a vacuum for subsequent use as laser targets and neutron generation is the subject of active research [40]. The developed methods allow us to analyze the thermodynamic state of such multicomponent mixtures.

\section{Methods}

One of the most common methods at an early stage of studying supercritical fluids' properties is molecular dynamics (MD), which is a bridge between experimentally observed macroscopic properties of a substance with microscopic ones. This approach has been previously used to study the Widom delta separating the region of liquid-like from gas-like SCF in the p-T diagram [53-55]. Molecular dynamics (MD) is a method used for numerical simulation of the system evolution in time by integrating the equations of motion of atoms or particles. The movements of atoms or particles are calculated based on classical mechanics. Within the framework of the MD, the forces of interatomic interaction are represented in the form of the classical potential forces (the gradient of the potential energy of the system). The interatomic potential primarily determines the MD. The solution of the classical equations of motion in the framework of the MD leads to a set of trajectories that consist of atomic positions $r$ and velocities $v$ (or as an equivalent of momenta $p$ ) as a function of time $t$ [56]. The thermodynamic parameters of the system are calculated as a function of these parameters. Since classical thermodynamics operates with continuous parameters formulated without considering the atomic (discrete) nature of matter, it does not connect thermodynamic quantities and atomic trajectories. Therefore, to retrieve TD parameters, it is necessary to use statistical thermodynamics (for equilibrium systems). The most used thermodynamic parameters are heat capacity at constant pressure $\left(C_{p}\right)$, isothermal compressibility $(\beta)$, and coefficient of thermal expansion $(\alpha)$. In the numerical simulation, the heat capacity can be defined as:

$$
C_{p}=\frac{H(p, T+\varepsilon)-H(p, T-\varepsilon)}{2 \varepsilon},
$$

where $H$ is enthalpy, $p$ is pressure, $T$ is temperature, $V$ is volume of system, and $\varepsilon$ is small deviation ( $0.5 \mathrm{~K}$ and 0.1 bar in our numerical simulation). Isothermal compressibility and coefficient of thermal expansion are calculated similarly.

$$
\begin{gathered}
\beta=-\frac{1}{V(p, T)} \frac{V(p+\varepsilon, T)-V(p-\varepsilon, T)}{2 \varepsilon}, \\
\alpha=\frac{1}{V(p, T)} \frac{V(p, T+\varepsilon)-V(p, T-\varepsilon)}{2 \varepsilon},
\end{gathered}
$$

In this work, we used the LAMMPS software package [56]. The simulation was carried out for about 100,000 atoms with periodic boundary conditions, the interatomic interaction was specified by the COMPASS potential [57]. We used sixth power mixing rules and a long-range VanderWaals "correction" to the energy and pressure. The molecules were constructed with a MOLTEMPLATE utility [58]. The ethanol molecule was constructed based on the following script [59] and $\mathrm{CO}_{2}$ molecule from the following example [60]. The potential is an ab initio "class II" potential based on a matched force potential, which is successfully used to simulate both organic and inorganic molecules even at high (up to 2000 bar) pressures [61]. The choice of this potential was motivated by the best agreement between the calculated and experimental Raman spectra. The modeling was carried out in several stages. At the first stage, the system was brought into thermodynamic equilibrium (the criterion was the invariability of the internal energy and enthalpy of the system in 100,000 steps). To do this, we consistently simulated a microcanonical ensemble with a Langevin thermostat [62] and a Berendsen barostat [63] (fix NVE + fix 
Langevin + fix pressure/bredson) with a step of 1 femtosecond (fs). The combination of the applied fixes gives the best agreement with tabular values of density and enthalpy for pure $\mathrm{CO}_{2}$ and ethanol. For the initial achievement of TD equilibrium for a given temperature and atmospheric pressure, 100 million steps were taken. Further, for pressures with a step of 5 bar for a given temperature, the system was sequentially brought to the TD in equilibrium (fix NVE + fix Langevin + fix pressure/bredson) in 10 million steps (step is $0.1 \mathrm{fs}$ ). Then, for averaging over 100 points, the system's density, volume, and enthalpy were calculated every 100,000 steps by built-in LAMMPS functions. Similar measurements were repeated for points on the $\mathrm{p}$ - $\mathrm{T}$ diagram at $0.1 \mathrm{bar}$ and $0.5 \mathrm{~K}$, respectively ( 4 points in total) for calculations by Equations (1)-(3). The Widom delta was determined as a region, where a maximum of fluctuations is reached [10], an extremum of the TD parameters is observed [17-19], and the maximum formation of clusters is achieved [32].

The applied numerical model was initially verified on pure $\mathrm{CO}_{2}$ and ethanol. The computed values of density and enthalpy were compared with tabular ones [64] (see Figure 9). Without vicinity of critical pressure, the calculated values are identical with tabular. In ethanol the calculated density and enthalpy for room conditions coincide with tabular data $\left(0.785 \mathrm{~kg} / \mathrm{m}^{3}, 38.5 \mathrm{~kJ} / \mathrm{mol}\right.$ and $0.789 \mathrm{~kg} / \mathrm{m}^{3}, 38.56 \mathrm{~kJ} / \mathrm{mol}$ respectively), the calculated critical point $(507 \mathrm{~K}, 67 \mathrm{bar})$ is close to tabular $(514 \mathrm{~K}, 63 \mathrm{bar})$. In critical point the difference between the calculated density/enthalpy with literature data $\left(0.276 \mathrm{~kg} / \mathrm{m}^{3}\right.$ and $15.75 \mathrm{~kJ} / \mathrm{mol})$ [65] is higher $\left(0.289 \mathrm{~kg} / \mathrm{m}^{3}\right.$ and $\left.13.38 \mathrm{~kJ} / \mathrm{mol}\right)$. The difference in the vicinity of the critical point is caused by slightly different values of critical parameters retrieved from molecular dynamics. Nevertheless, the observed differences do not change the effects of clustering qualitatively, only modify the Widom delta's boundary.
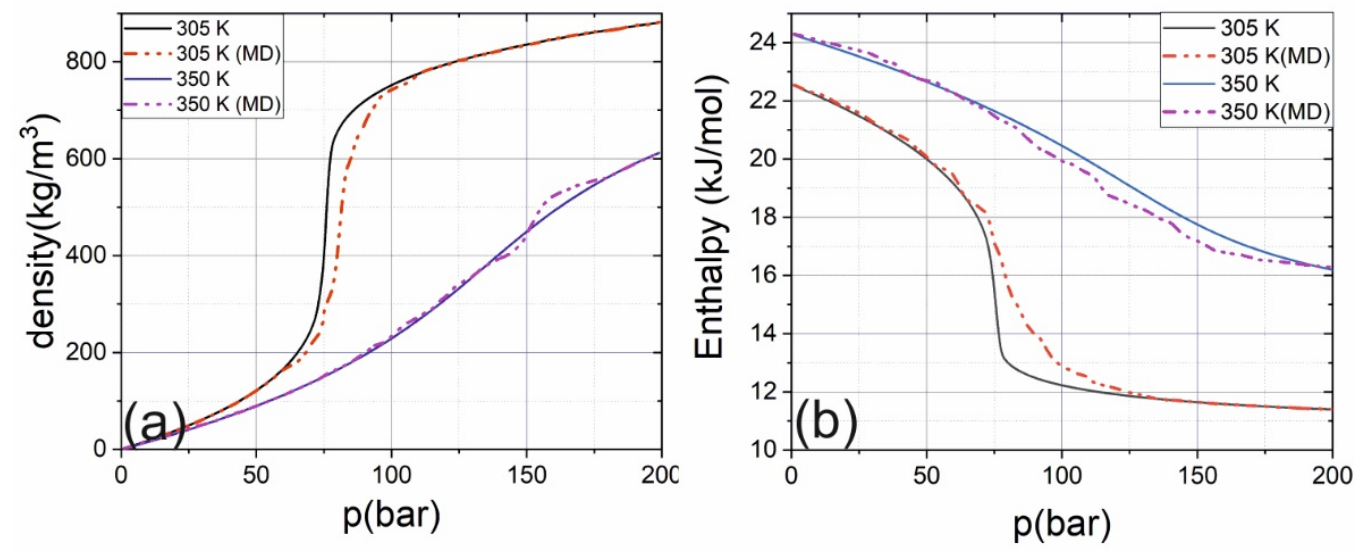

Figure 9. Dependence of computed (dash-dot lines) and tabular (solid lines) values of density (a) and enthalpy (b) on pressure for $\mathrm{CO}_{2}$ different temperatures.

\section{Conclusions}

We analyze the structure of a mixture containing carbon dioxide with ethanol ( $25 \%$ of the mixture) and the behavior of density, its fluctuations, enthalpy, and thermodynamic parameters in a wide range of temperatures and pressures including the supercritical state using molecular dynamics. It was revealed that there are three regions of significant (doubled compared with pure $\mathrm{CO}_{2}$ ) increase in density fluctuations, which are identified as Widom deltas. In these regions, the disintegration of the supercluster was observed, accompanied by a significant decrease in the number of molecules in it (more than three times), as well as an increase in the number of large linear mixed clusters (10-50 molecules). It has been shown that anomalous behavior of such parameters as enthalpy and isobaric heat capacity is a consequence of an increase in the number of medium-sized clusters and the decay of a supercluster corresponding to the liquid state of matter. It would be expected that the appearance of new Widom delta in the solvent mixture would lead to an increase in the reactivity of the complex solution. We believe that the complex mixtures can open a new route for the emergency of novel materials or efficient solvents. We expected that 
the additional information about microstructure of mixtures in such a promising region as Widom delta will find applications in various areas of science and technology. For example, increase in density fluctuations could increase the material removal rate during laser ablation; "the cluster formation in the Widom delta will increase the nonlinearity of the medium, which is interesting for generating a supercontinuum or for self-compression of ultrafast laser pulses, and the possible increase in the diffusivity of the mixture for the extraction of various organic and inorganic materials is also significant.

Author Contributions: Conceptualization, E.I.M. and V.M.G.; methodology, E.I.M.; software, E.I.M.; validation, E.I.M.; formal analysis, E.I.M., A.P.S. and V.M.G.; investigation, E.I.M., A.P.S. and V.M.G.; resources, A.P.S.; data curation, E.I.M.; writing—original draft preparation, E.I.M.; writing—review and editing, A.P.S. and V.M.G.; visualization, E.I.M.; supervision, V.M.G.; project administration, A.P.S.; funding acquisition, E.I.M. and V.M.G. All authors have read and agreed to the published version of the manuscript.

Funding: This work was supported by RFBR projects: 19-32-60072 (modeling of Widom delta), 18-29-06035 (modeling of binary mixtures), and 18-29-06056 (modeling of density fluctuations), and by the Ministry of Science and Higher Education within the State assignment FSRC «Crystallography and Photonics» RAS (cluster formation).

Institutional Review Board Statement: Not applicable.

Informed Consent Statement: Not applicable.

Data Availability Statement: The data presented in this study are available on request from the corresponding author. The data are not publicly available due to privacy.

Acknowledgments: The authors are grateful to V.A. Aleshkevich and A.V. Lazarev for a fruitful discussion of the results and advice on preparing the article.

Conflicts of Interest: The authors declare no conflict of interest. The funders had no role in the design of the study; in the collection, analyses, or interpretation of data; in the writing of the manuscript, or in the decision to publish the results.

\section{References}

1. Brunner, G. Applications of Supercritical Fluids. Annu. Rev. Chem. Biomol. Eng. 2010, 1, 321-342. [CrossRef] [PubMed]

2. Bellan, J. Supercritical (and subcritical) fluid behavior and modeling: Drops, streams, shear and mixing layers, jets and sprays. Prog. Energy Combust. Sci. 2000, 26, 329-366. [CrossRef]

3. MacHmudah, S.; Wahyudiono; Kuwahara, Y.; Sasaki, M.; Goto, M. Nano-structured particles production using pulsed laser ablation of gold plate in supercritical $\mathrm{CO}_{2}$. J. Supercrit. Fluids 2011, 60, 63-68. [CrossRef]

4. Byrappa, K.; Ohara, S.; Adschiri, T. Nanoparticles synthesis using supercritical fluid technology-Towards biomedical applications. Adv. Drug Deliv. Rev. 2008, 60, 299-327. [CrossRef] [PubMed]

5. Taylor, L.T. Supercritical fluid chromatography. Anal. Chem. 2010, 82, 4925-4935. [CrossRef]

6. Gorelli, F.A.; Bryk, T.; Krisch, M.; Ruocco, G.; Santoro, M.; Scopigno, T. Dynamics and Thermodynamics beyond the critical point. Sci. Rep. 2013, 3, 1203. [CrossRef] [PubMed]

7. Simeoni, G.G.; Bryk, T.; Gorelli, F.A.; Krisch, M.; Ruocco, G.; Santoro, M.; Scopigno, T. The Widom line as the crossover between liquid-like and gas-like behaviour in supercritical fluids. Nat. Phys. 2010, 6, 503-507. [CrossRef]

8. Whitesides, G.M. The origins and the future of microfluidics. Nature 2006, 442, 368-373. [CrossRef]

9. McMillan, P.F.; Stanley, H.E. Fluid phases: Going supercritical. Nat. Phys. 2010, 6, 479-480. [CrossRef]

10. Nishikawa, K.; Tanaka, I. Correlation lengths and density fluctuations in supercritical states of carbon dioxide. Chem. Phys. Lett. 1995, 244, 149-152. [CrossRef]

11. Brazhkin, V.V.; Lyapin, A.G.; Ryzhov, V.N.; Trachenko, K.; Fomin, Y.D.; Tsiok, E.N. Where is the supercritical fluid on the phase diagram? Physics-Uspekhi 2012, 55, 1061-1079. [CrossRef]

12. Sedunov, B. The Analysis of the Equilibrium Cluster Structure in Supercritical Carbon Dioxide. Am. J. Anal. Chem. 2012, 3, 899-904. [CrossRef]

13. Gorelli, F.; Santoro, M.; Scopigno, T.; Krisch, M.; Ruocco, G. Liquidlike behavior of supercritical fluids. Phys. Rev. Lett. 2006, 97, 15-18. [CrossRef]

14. Ha, M.Y.; Yoon, T.J.; Tlusty, T.; Jho, Y.; Lee, W.B. Widom Delta of Supercritical Gas-Liquid Coexistence. J. Phys. Chem. Lett. 2018, 9 , 1734-1738. [CrossRef]

15. Sciortino, F.; Poole, P.H.; Essmann, U.; Stanley, H.E. Line of compressibility maxima in the phase diagram of supercooled water. Phys. Rev. E-Stat. Phys. Plasmas Fluids Relat. Interdiscip. Top. 1997, 55, 727-737. [CrossRef] 
16. May, H.O.; Mausbach, P. Riemannian geometry study of vapor-liquid phase equilibria and supercritical behavior of the LennardJones fluid. Phys. Rev. E-Stat. Nonlinear Soft Matter Phys. 2012, 85, 1-9. [CrossRef]

17. Liu, L.; Chen, S.H.; Faraone, A.; Yen, C.W.; Mou, C.Y. Pressure dependence of fragile-to-strong transition and a possible second critical point in supercooled confined water. Phys. Rev. Lett. 2005, 95, 1-4. [CrossRef] [PubMed]

18. Santoro, M.; Gorelli, F.A. Structural changes in supercritical fluids at high pressures. Phys. Rev. B-Condens. Matter Mater. Phys. 2008, 77, 1-4. [CrossRef]

19. Banuti, D.T. Crossing the Widom-line-Supercritical pseudo-boiling. J. Supercrit. Fluids 2015, 98, 12-16. [CrossRef]

20. Ruppeiner, G.; Sahay, A.; Sarkar, T.; Sengupta, G. Thermodynamic geometry, phase transitions, and the Widom line. Phys. Rev. E-Stat. Nonlinear Soft Matter Phys. 2012, 86, 3-6. [CrossRef]

21. Raju, M.; Banuti, D.T.; Ma, P.C.; Ihme, M. Widom lines in binary mixtures of supercritical fluids. Sci. Rep. 2017, 7, 3027. [CrossRef] [PubMed]

22. Imre, A.R.; Ramboz, C.; Deiters, U.K.; Kraska, T. Anomalous fluid properties of carbon dioxide in the supercritical region: Application to geological $\mathrm{CO}_{2}$ storage and related hazards. Environ. Earth Sci. 2015, 73, 4373-4384. [CrossRef]

23. Imre, A.R.; Házi, G.; Horváth, Á.; Maráczy, C.; Mazur, V.; Artemenko, S. The effect of low-concentration inorganic materials on the behaviour of supercritical water. Nucl. Eng. Des. 2011, 241, 296-300. [CrossRef]

24. Nishikawa, K.; Morita, T. Inhomogeneity of molecular distribution in supercritical fluids Inhomogeneity of molecular distribution in supercritical fluids. Chem. Phys. Lett. 2000, 316, 238-242. [CrossRef]

25. Mareev, E.; Aleshkevich, V.; Potemkin, F.; Bagratashvili, V.; Minaev, N.; Gordienko, V. Anomalous behavior of nonlinear refractive indexes of $\mathrm{CO}_{2}$ and Xe in supercritical states. Opt. Express 2018, 26, 13229-13238. [CrossRef] [PubMed]

26. Mareev, E.I.; Aleshkevich, V.A.; Potemkin, F.V.; Minaev, N.V.; Gordienko, V.M. Molecular Refraction and Nonlinear Refractive Index of Supercritical Carbon Dioxide under Clustering Conditions. Russ. J. Phys. Chem. B 2019, 13, 1-6. [CrossRef]

27. Bolmatov, D.; Zav'Yalov, D.; Gao, M.; Zhernenkov, M. Structural evolution of supercritical $\mathrm{CO}_{2}$ across the Frenkel line. J. Phys. Chem. Lett. 2014, 5, 2785-2790. [CrossRef]

28. Fomin, D.; Ryzhov, V.N.; Tsiok, E.N.; Brazhkin, V.V.; Trachenko, K. Thermodynamics and Widom lines in supercritical carbon dioxide. Phys. Rev. E 2015, 91, 022111. [CrossRef]

29. Brazhkin, V.V.; Fomin, Y.D.; Ryzhov, V.N.; Tareyeva, E.E.; Tsiok, E.N. True Widom line for a square-well system. Phys. Rev. E-Stat. Nonlinear Soft Matter Phys. 2014, 89, 1-6. [CrossRef]

30. Brazhkin, V.V.; Fomin, Y.D.; Lyapin, A.G.; Ryzhov, V.N.; Trachenko, K. Two liquid states of matter: A dynamic line on a phase diagram. Phys. Rev. E-Stat. Nonlinear Soft Matter Phys. 2012, 85, 1-12. [CrossRef] [PubMed]

31. Imre, A.R.; Deiters, U.K.; Kraska, T.; Tiselj, I. The pseudocritical regions for supercritical water. Nucl. Eng. Des. 2012, 252, 179-183. [CrossRef]

32. Mareev, E.; Semenov, T.; Lazarev, A.; Minaev, N.; Sviridov, A.; Potemkin, F.; Gordienko, V. Optical Diagnostics of Supercritical $\mathrm{CO}_{2}$ and $\mathrm{CO}_{2}$-Ethanol Mixture in the Widom Delta. Molecules 2020, 25, 5424. [CrossRef] [PubMed]

33. Grijó, D.R.; Vieitez Osorio, I.A.; Cardozo-Filho, L. Supercritical extraction strategies using $\mathrm{CO}_{2}$ and ethanol to obtain cannabinoid compounds from Cannabis hybrid flowers. J. $\mathrm{CO}_{2}$ Util. 2018, 28, 174-180. [CrossRef]

34. Sarker, M.Z.I.; Selamat, J.; Habib, A.S.M.A.; Ferdosh, S.; Akanda, M.J.H.; Jaffri, J.M. Optimization of supercritical $\mathrm{CO}_{2}$ extraction of fish oil from viscera of African Catfish (Clarias gariepinus). Int. J. Mol. Sci. 2012, 13, 11312-11322. [CrossRef]

35. McHugh, M.A.; Krukonis, V.J. Supercritical Fluid Extraction: Principles and Practice; Butterworth-Heinemann: Oxford, UK, 1994; ISBN 9780080518176.

36. Reddy, V.; Saharay, M. Solubility of Caffeine in Supercritical $\mathrm{CO}_{2}$ : A Molecular Dynamics Simulation Study. J. Phys. Chem. B 2019, 123, 9685-9691. [CrossRef]

37. Woźniak, L.; Marszalek, K.; Skapska, S.; Jedrzejczak, R. The application of supercritical carbon dioxide and ethanol for the extraction of phenolic compounds from chokeberry pomace. Appl. Sci. 2017, 7, 322. [CrossRef]

38. Artemenko, S.; Krijgsman, P.; Mazur, V. The Widom line for supercritical fluids. J. Mol. Liq. 2017, 238, 122-128. [CrossRef]

39. Braeuer, A.; Dowy, S.; Leipertz, A.; Schatz, R.; Schluecker, E. Injection of ethanol into supercritical $\mathrm{CO}_{2}$ : Determination of mole fraction and phase state using linear Raman scattering. Opt. Express 2007, 15, 8377. [CrossRef]

40. Lalanne, P.; Tassaing, T.; Danten, Y.; Cansell, F.; Tucker, S.C.; Besnard, M. $\mathrm{CO}_{2}$-Ethanol Interaction Studied by Vibrational Spectroscopy in Supercritical CO $\mathrm{CO}_{2}$. J. Phys. Chem. A 2004, 108, 2617-2624. [CrossRef]

41. Cordray, D.R.; Izatt, R.M.; Christensen, J.J.; Oscarson, J.L. Dortmund Data Bank. Available online: http:// www.ddbst.com/en/ EED/HE/HEEthanol\%3BCarbondioxide.php (accessed on 7 December 2020).

42. Day, C.Y.; Chang, C.J.; Chen, C.Y. Phase equilibrium of ethanol $+\mathrm{CO}_{2}$ and acetone $+\mathrm{CO}_{2}$ at elevated pressures. J. Chem. Eng. Data 1996, 41, 839-843. [CrossRef]

43. Span, R.; Wagner, W. NIST Database. Available online: http://webbook.nist.gov/ (accessed on 9 September 2021).

44. Yoon, T.J.; Ha, M.Y.; Lee, W.B.; Lee, Y.W. A corresponding-state framework for the structural transition of supercritical fluids across the Widom delta. J. Chem. Phys. 2019, 150, 154503. [CrossRef]

45. Rycroft, C.H. VORO++: A three-dimensional Voronoi cell library in C++. Chaos 2009, 19, 41111. [CrossRef]

46. Gorbaty, Y.; Bondarenko, G.V. Transition of liquid water to the supercritical state. J. Mol. Liq. 2017, 239, 5-9. [CrossRef]

47. Nishikawa, K.; Tanaka, I.; Amemiya, Y. Small-Angle X-ray Scattering Study of Supercritical Carbon Dioxide. J. Phys. Chem. 1996, 100, 418-421. [CrossRef] 
48. Arakcheev, V.G.; Bagratashvili, V.N.; Valeev, A.A.B.; Gordienko, V.M.; Kireev, V.V.; Morozov, V.B.; Olenin, A.N.; Popov, V.K.; Tunkin, V.G.E.; Yakovlev, D.V. CARS spectroscopy of carbon dioxide in the critical point vicinity. Quantum Electron. 2007, 34, 86-90. [CrossRef]

49. Morita, T.; Nishikawa, K.; Takematsu, M.; Iida, H.; Furutaka, S. Structure study of supercritical $\mathrm{CO}_{2}$ near high-order phase transition line by X-ray diffraction. J. Phys. Chem. B 1997, 101, 7158-7162. [CrossRef]

50. Subramanlamr, B.; McHugh, M.A. Reactions in Supercritical Fluids-A Review. Ind. Eng. Chem. Process Des. Dev. 1986, 25, 1-12. [CrossRef]

51. Oparin, R.D.; Krestyaninov, M.A.; Vorobyev, E.A.; Pokrovskiy, O.I.; Parenago, O.O.; Kiselev, M.G. An insight into possibility of chemical reaction between dense carbon dioxide and methanol. J. Mol. Liq. 2017, 239, 83-91. [CrossRef]

52. Lazarev, A.V.; Tatarenko, K.A. Gas dynamic model of the expansion of a supercritical carbon dioxide pulse jet: A self-similar solution. Russ. J. Phys. Chem. B 2016, 10, 1248-1255. [CrossRef]

53. Strong, S.E.; Shi, L.; Skinner, J.L. Percolation in supercritical water: Do the Widom and percolation lines coincide? J. Chem. Phys. 2018, 149, 084504. [CrossRef]

54. Guevara-Carrion, G.; Ancherbak, S.; Mialdun, A.; Vrabec, J.; Shevtsova, V. Diffusion of methane in supercritical carbon dioxide across the Widom line. Sci. Rep. 2019, 9, 8466. [CrossRef]

55. Bolmatov, D.; Zhernenkov, M.; Zav'yalov, D.; Tkachev, S.N.; Cunsolo, A.; Cai, Y.Q. The Frenkel Line: A direct experimental evidence for the new thermodynamic boundary. Sci. Rep. 2015, 5, 15850. [CrossRef]

56. Plimpton, S. Fast Parallel Algorithms for Short-Range Molecular Dynamics. J. Comput. Phys. 1995, 117, 1-42. [CrossRef]

57. COMPASS. Force Field Manual; COMPASS: Calgary, AB, Canada, 1998; pp. 1-48.

58. Jewett, A.I.; Stelter, D.; Lambert, J.; Saladi, S.M.; Roscioni, O.M.; Ricci, M.; Autin, L.; Maritan, M.; Bashusqeh, S.M.; Keyes, T.; et al. Moltemplate: A Tool for Coarse-Grained Modeling of Complex Biological Matter and Soft Condensed Matter Physics. J. Mol. Biol. 2021, 433, 166841. [CrossRef]

59. Jewettaji, A. Ethanol Molecule. Available online: https://github.com/jewettaij/moltemplate/blob/master/examples/all_atom/ force_field_OPLSAA/waterTIP5P+ethanol/moltemplate_files/ethanol.lt (accessed on 9 September 2021).

60. Kohlmeyer, A. $\mathrm{CO}_{2}$ Molecule. Available online: https://github.com/lammps/lammps/blob/master/examples/gcmc/in.gcmc. co2 (accessed on 9 September 2021).

61. Rigby, D. Fluid density predictions using the COMPASS force field. Fluid Phase Equilib. 2004, 217, 77-87. [CrossRef]

62. Schneider, T.; Stoll, E. Molecular-dynamics study of a three-dimensional one-component model for distortive phase transitions. Phys. Rev. B 1978, 17, 1302-1322. [CrossRef]

63. Berendsen, H.J.C.; Postma, J.P.M.; Van Gunsteren, W.F.; Dinola, A.; Haak, J.R. Molecular dynamics with coupling to an external bath. J. Chem. Phys. 1984, 81, 3684-3690. [CrossRef]

64. Goos, E.; Riedel, U.; Zhao, L.; Blum, L. Phase diagrams of $\mathrm{CO}_{2}$ and $\mathrm{CO}_{2}-\mathrm{N} 2$ gas mixtures and their application in compression processes. Energy Procedia 2011, 4, 3778-3785. [CrossRef]

65. Marcus, Y. Extraction by subcritical and supercriticalwater, methanol, ethanol and their mixtures. Separations 2018, 5, 4. [CrossRef] 